\title{
Evaluating the Frequency of Sciatica on Magnetic Resonance Imaging in Patients with Lower Back Pain
}

\author{
Muhammad Ahmad Naeem* \\ Lecturer, The University of Lahore, Gujrat Campus, University of Lahore, Adjacent Chenab bridge, Gujrat, \\ Pakistan \\ Rana Muhammad Athar Azeem Shams \\ Lecturer, Superior College Lahore, University Campus, 17-KM Raiwind Road, Kot Arain, Lahore, Pakistan
}

Rabia Razzaq

Lecturer, Imperial College of Business Studies, Shakam Industries, Lahore, Pakistan

Zain Ul Hassan

Assistant Professor, The University of Lahore, Lahore Campus, 1-KM Defence Road, Lahore, Pakistan

Rehan Asfar

Lecturer, The University of Lahore, Gujrat Campus

University of Lahore, Adjacent Chenab bridge, Gujrat, Pakistan

Tuseef Ahmed

Lecturer, The University of Lahore, Lahore Campus, 1-KM Defence Road, Lahore, Pakistan

Muhammad Uzair

Lecturer, The University of Lahore, Lahore Campus, 1-KM Defence Road, Lahore, Pakistan

Sybil Rose

Lecturer, Superior College Lahore, University Campus, 17-KM Raiwind Road, Kot Arain, Lahore, Pakistan

\begin{abstract}
Sciatica refers to pain affecting the back, hip, and outer side of the leg, caused by compression of a spinal nerve root in the lower back often owing to degeneration of an intervertebral disc. The objective of this study was to determine the frequency of sciatica in patients with lower back pain using magnetic resonance imaging (MRI). Data was collected by filling the predesign questionnaire. Information for filling the questionnaire was taken by reports of MRI scan for backache. Patients were presented with multiple symptoms out of which $4(5.6 \%)$ patients presented with history of trauma. 37 (59.7\%) patients represented with the history pain radiating to one or both legs. 27 (43.50\%) patients showed disc bulge. 40 (64.50\%) patients showed stenosis in nerve root (L4-S1) and $40(64.50 \%)$ patients were diagnosed with sciatica. Careful assessment of risk factors in patients with lower back pain using MRI could help in prevention of several spinal diseases. Being an important diagnostic tool in diagnosis of sciatica MRI may provide significant prognostic information of the disease.
\end{abstract}

Keywords: Stenosis, Disc Bulge.

DOI: $10.7176 / \mathrm{JHMN} / 80-03$

Publication date:September $30^{\text {th }} 2020$

\section{Introduction}

Low back pain (LBP) is a common disorder involving the muscles, nerves, and bones of the back. Pain can vary from a dull constant ache to a sudden sharp feeling. Low back pain may be classified by duration as acute (pain lasting less than 6 weeks), sub-chronic ( 6 to 12 weeks), or chronic (more than 12 weeks). The condition may be further classified by the underlying cause as either mechanical, non-mechanical, or referred pain. Some lower back pain is caused by damaged intervertebral discs. Sciatica refers to pain affecting the back, hip, and outer side of the leg, caused by compression of a spinal nerve root in the lower back often owing to degeneration of an intervertebral disc.

1.1 Spinal disc Herniation

A spinal disc herniation, commonly referred to as a slipped disc, can happen when unbalanced mechanical pressures substantially deform the annulus fibrosis, allowing part of the nucleus to obtrude. These events can occur during peak physical performance, during traumas, or as a result of chronic deterioration, typically characterized by poor posture. Both the deformed annulus and the gel-like material of the nucleus pulposus can 
be forced laterally, or anteriorly, distorting local muscle function, and putting pressure on the nearby nerve. This can give the symptoms typical of nerve root entrapment. These symptoms can vary between paresthesia, numbness, chronic and/or acute pain, either locally or along the dermatome served by the entrapped nerve, loss of muscle tone and decreased homeostatic performance. The disc is not physically slipped; it bulges, usually in just one direction. Another kind of herniation, of the nucleus pulposus, can also happen as a result of the formation of Schmorl's nodes on the intervertebral disc and is referred to as vertical disc herniation.

\subsection{Disc Degeneration}

Before age 40 approximately $25 \%$ of people show evidence of disc degeneration at one or more levels. Beyond age 40 , more than $60 \%$ of people show evidence of disc degeneration at one or more levels on magnetic resonance imaging (MRI). One effect of ageing and disc degeneration is that the nucleus pulposus begins to dehydrate and the concentration of proteoglycans in the matrix decreases, thus limiting the ability of the disc to absorb shock. This general shrinking of disc size is partially responsible for the common decrease in height as human's age. The annulus fibrosis also becomes weaker with age and has an increased risk of tearing. In addition, the cartilage endplates begin thinning, fissures begin to form, and there is sclerosis of the subchondral bone. As the fissures are formed in the annulus fibrosis due to osteoarthritic bones or degeneration in general, the inner nucleus pulposus can seep out and put pressure on any number of vertebral nerves. A herniated disc can cause mild to severe pain such as sciatica and treatment for herniated discs range from physical therapy to surgery. Other degeneration of the vertebral column includes diffuse idiopathic skeletal hyperostosis (DISH) which is the calcification or ossification of the ligaments surrounding the vertebrae. This degeneration causes stiffness and sometimes even curvature in the lumbar and thoraco-lumbar spinal region.

\subsection{Scoliosis}

While this may not cause pain in some people, in others it may cause chronic pain. Other spinal disorders can affect the morphology of intervertebral discs. For example, patients with scoliosis commonly have calcium deposits (ectopic calcification) in the cartilage endplate and sometimes in the disc itself. Herniated discs are also found to have a higher degree of cellular senescence than non-herniated discs. In addition to scoliosis, which is the lateral 'S' curvature of the spine, the fused vertebrae can also experience other abnormalities such as kyphosis (hunchback) which shows in old age, or lordosis (swayback), which is often present in pregnancy and obesity.

\subsection{Intervertebral disc space}

The intervertebral disc space is typically defined on an X-ray photograph as the space between adjacent vertebrae. In healthy patients, this corresponds to the size of the intervertebral disc. The size of the space can be altered in pathological conditions such as discitis (infection of the intervertebral disc).

Magnetic resonance imaging is a medical imaging technique used in radiology to form pictures of the anatomy and the physiological processes of the body in both health and disease. MRI scanners use strong magnetic fields, electric field gradients, and radio waves to generate images of the organs in the body. MRI does not involve $\mathrm{X}$-rays and the use of ionizing radiation.

\section{Methodology}

This was retrospective Observational study. Sample of 62 patients was taken in this study. This study was conducted at Radiology Department Al-Razi Health Care, Lahore. All males and females presenting with sciatica and backache were included in this study. Patients with metal implants and Emergency cases were not included in this study. This study was completed in 6 months. Performa was filled according to the collected information of inclusive patients. The inclusive patients underwent magnetic resonance imaging of the lumber spine. The reports were collected. Data was collected by filling the predesign Performa. Information for filling the Performa was taken by the reports of Magnetic Resonance Imaging scan for lower back pain. The data was entered and analyzed using IBM-SPSS (Statistical package for social sciences). All quantitative data was discussed in mean and standard deviation. Whereas categorical variables in form of frequency and percentage charts were used to display the data.

\section{Results}

There were 62 participants in the study with the age group of 20- 80 years, the maximum number of patients were from the age group 25-55 years. The mean age was 41.6 years. The mean and standard deviation were $41.6 \pm 13.1$ years. Twenty-nine patients $(46.8 \%)$ were female and thirty-three $(53.2 \%)$ were male. The presenting complaints were with multiple symptoms $4(6.4 \%)$ patients present with history of trauma. $37(59.7 \%)$ patients represent with history of pain radiating to legs. $27(43.50 \%)$ patients showed disc bulge and $40(64.50 \%)$ patients showed stenosis in nerve root as a cause of sciatica and $40(64.50 \%)$ patients showed sciatica. (Table 1) (Figure 1) 


\section{Discussion}

Shrinuvasan S and Chidambaram R conducted a study on Evaluation of low back pain. The age of the patient ranged from 21 to 68 years with an average of 41.3 years. Back pain was commonly observed in the third to fifth decade. The common causes for back pain are disc herniation (disc bulge - 35.3\%, disc protrusion - 39.6\%, disc extrusion $-7.2 \%$ ) accounting to $82.1 \%$, followed by normal study $(10.2 \%$ ), vertebral collapse (traumatic $-2.1 \%$, osteoporotic $-1.7 \%)$, infections $(2.1 \%)$, and neoplasm (1.7\%). MRI provides valuable information regarding the underlying causes of LBP, especially in disc and marrow pathology.

According to our study a total of $62(100 \%)$ patients presents with multiple symptoms out of which $4(6.4 \%)$ patients present with history of trauma.37 (59.7\%) patients represent with the history pain radiating to legs.27 $(43.50 \%)$ patients showed disc bulge and $40(64.50 \%)$ patients showed stenosis in nerve root as a cause of sciatica and $40(64.50 \%)$ patients showed sciatica.

\section{Conclusion}

From the above analysis, we can conclude that sacral plexus stenosis is the main cause of sciatica, and its symptoms include lower back pain and pain radiate to one or both legs. And magnetic resonance imaging is proved to a useful tool in diagnosis of sciatica.

\section{Limitations}

Sample size should be large.

\section{References}

Carrino, J. A., Campbell Jr, P. D., Lin, D. C., Morrison, W. B., Schweitzer, M. E., Flanders, A. E., Eng, J. \& Vaccaro, A. R. 2011. Effect of Spinal Segment Variants on Numbering Vertebral Levels at Lumbar MR Imaging. Radiology, 259, 196-202.

Chaurasia, B. D. \& Goswami, H. 1975. Functional Asymmetry in the Face. Cells Tissues Organs, 91, 154-160.

Filler, A. G., Haynes, J., Jordan, S. E., Prager, J., Villablanca, J. P., Farahani, K., Mcbride, D. Q., Tsuruda, J. S., Morisoli, B. \& Batzdorf, U. 2005. Sciatica of Nondisc Origin and Piriformis Syndrome: Diagnosis by Magnetic Resonance Neurography and Interventional Magnetic Resonance Imaging with Outcome Study of Resulting Treatment. Journal of Neurosurgery: Spine, 2, 99-115.

Hall, j. E. 2015. Guyton and Hall Textbook of Medical Physiology E-book, Elsevier Health Sciences.

Mccarron, R. F., Wimpee, M. W., Hudkins, P. G. \& Laros, G. S. 1987. The Inflammatory Effect of Nucleus Pulposus: A Possible Element in the Pathogenesis of Low-Back Pain. Spine, 12, 760-764.

Omarker, K. \& Myers, R. R. 1998. Pathogenesis of Sciatic Pain: Role of Herniated Nucleus Pulposus and Deformation of Spinal Nerve Root and Dorsal Root Ganglion. Pain, 78, 99-105.

Snell, r. S. 2011. Clinical Anatomy by Regions, Lippincott Williams \& Wilkins. Waugh, A. \& Grant, A. 2010. Ross \& Wilson Anatomy and Physiology in Health and Illness E-book, Elsevier Health Sciences.

Wiltshire, M., Pendry, J., Young, I., Larkman, D., Gilderdale, D. \& Hajnal, J. 2001. Microstructured Magnetic Materials for RF Flux Guides in Magnetic Resonance Imaging. Science, 291, 849-851.

\section{Notes}

Note 1. A data of 62 patients was taken, that underwent magnetic resonance imaging tests during the study period. All the cases meet the inclusion criteria. The mean age was $41.6 \pm 13.1$ years. 


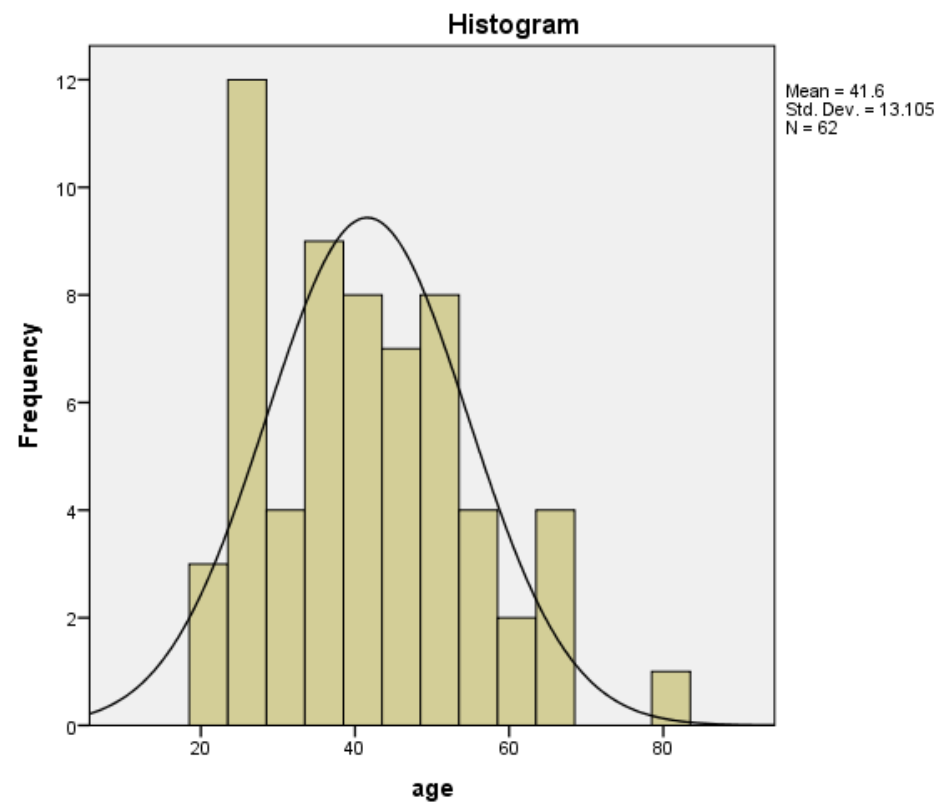

Graph 1: Mean age of patients

Note 2. This is a bar chart showing gender distribution of patients. Female (46.8\%) and males (53.2\%).

Gender

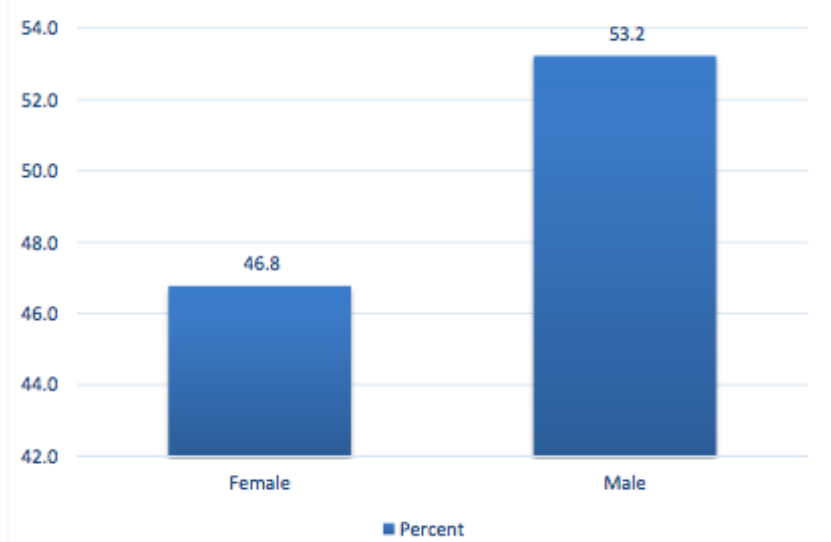

Graph 2: Bar Chart showing Gender Distribution of Patients

\begin{tabular}{|c|c|c|c|}
\hline \multicolumn{2}{|c|}{ Clinical History Parameters } & Frequency & Percentage \\
\hline \multirow{2}{*}{ Numbness } & Yes & 14 & $22.6 \%$ \\
& No & 48 & $77.4 \%$ \\
\hline \multirow{2}{*}{ Trauma } & Yes & 4 & $6.5 \%$ \\
& No & 58 & $93.5 \%$ \\
\hline \multirow{2}{*}{ Pain Radiating to legs } & Yes & 37 & $59.7 \%$ \\
& No & 25 & $40.3 \%$ \\
\hline
\end{tabular}

Table 1. Table showing clinical history of inclusive patients 


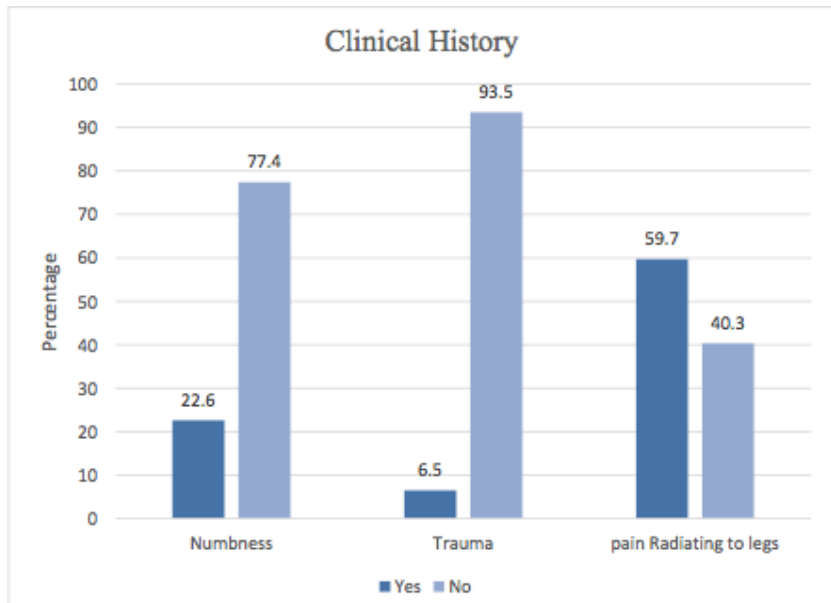

Graph 3: Bar Chart showing percentage of clinical history of inclusive patients

\begin{tabular}{|c|c|c|c|}
\hline \multicolumn{2}{|c|}{ MRI Diagnosis } & Frequency & Percentage \\
\hline \multirow{2}{*}{ Sciatica } & Yes & 40 & $64.5 \%$ \\
& No & 22 & $35.5 \%$ \\
\hline
\end{tabular}

Table 2. Table showing percentage of sciatica

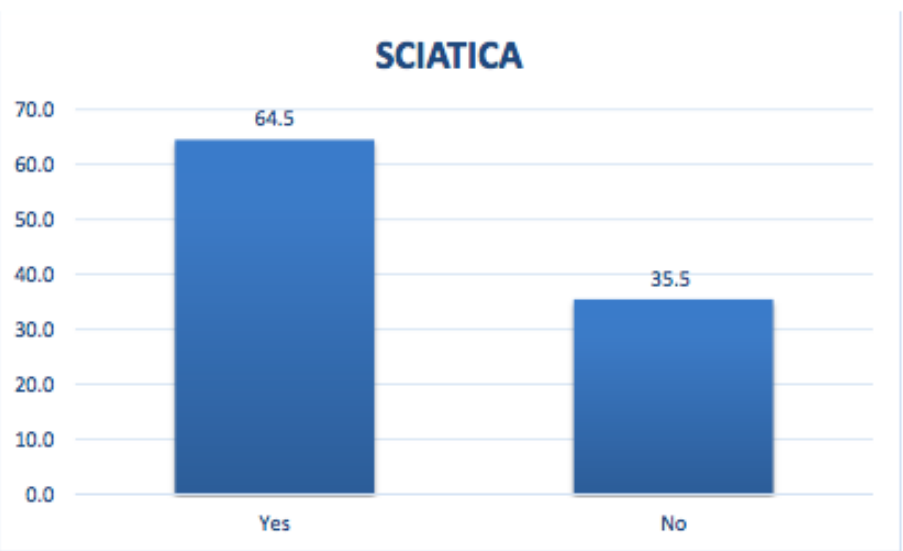

Graph 4: Bar Chart showing percentage of sciatica in patients

\begin{tabular}{|c|c|c|c|}
\hline \multicolumn{2}{|c|}{ Differential Diagnosis } & Frequency & Percentage \\
\hline \multirow{2}{*}{ Disc Bulge } & Yes & 27 & $43.50 \%$ \\
& No & 35 & $56.50 \%$ \\
\hline \multirow{2}{*}{ Stenosis } & Yes & 40 & $64.50 \%$ \\
& No & 22 & $35.50 \%$ \\
\hline
\end{tabular}

Table 3. Table showing differential diagnosis for causes

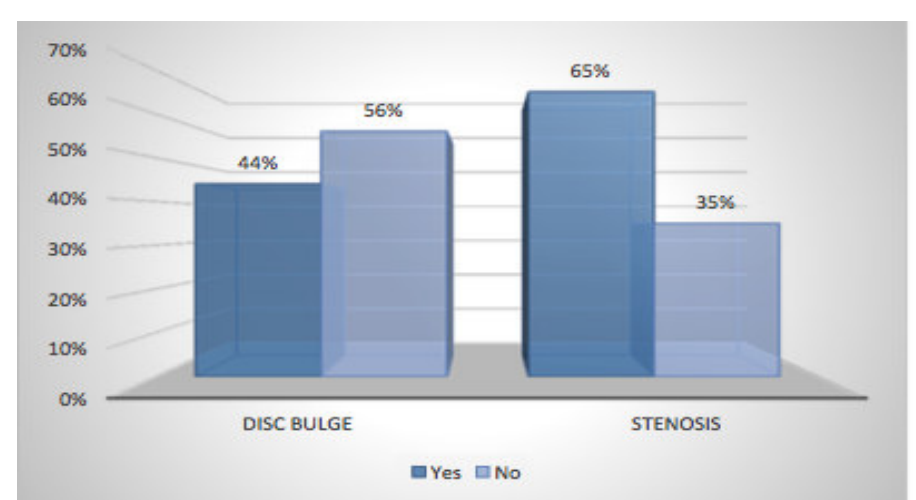

Graph 5: Bar Chart showing differential diagnosis of lower back pain 